Dr. Ma Wen-Gan

Department of Modern Physics

Univ. of Sci. and Tech. of China

Hefei, Anhui 230027, P. R. China, E-mail: mawg@ustc.edu.cn

Fax:(+86)-551-3601164

Tel:(+86)-551-3607034

November 4, 2018

Journal Supervisor

Physical Review D

American Institute of Physics

500 Sunnyside Boulevard

Woodbury, NY 11797-2999

Dear Editor,

This is the cover letter for the resubmission of our manuscript named 'Pair production of neutralinos via gluon-gluon collisions '(coded DN7258), written by Jiang Yi, Ma Wen-Gan, Han Liang, Yu Zeng-Hui and H. Pietschmann, to Physical Review D1 as regular article.

We did some modifications in our manuscript according to the referee's suggestions, 1. For the language problems: (1) The misspelled words are changed. (2) The symbol ' $+\mathrm{X}$ ' is added for the gg parton states in the subtitle of section 2. (3) The bar over the sum symbol in formula (2.9) is changed, and the symbol for the luminosity in formula (2.10) and (2.11) is adjusted.

2. For the more serious shortcomings: (1) The typing error in neutralino mass matrix (2.2) is corrected. (2) The argument about divergences on page 5 is omitted. The word 'evolution' is substituted by the word 'calculation'. (3) The illustration of 'the exchange of incoming gluons diragrams' is added. (4) The argument using Furry's theorem is changed. (5) The discussion on the Higgsino-like neutralinos pair production is added in the text and a relevant figure named 'Fig.(3)' is also added. (6) The references of renormalization group routine we used to calculate our low energy scenario from mSUGRA and the width of the intermediate Higgs bosons are added in section 3. (7) Some discussions of the curves about the cross section of 
neutralino pair production as the function of $\tan \beta$ are added.

3. For the additional questions: We use the newer sets of MRST of gluon densities to re-calculate the cross section and we found the newer results are only very little different between the MRST (mode 2) scheme and the older MRS (set G) scheme. This is addressed at the end of section 3. We also add the discussion of the uncertainty of scale variation in the text and add a figure named 'Fig.(2)'.

We are very grateful for the referee's useful suggestions.

With our best regards.

Sincerely yours,

Ma Wen-Gan 
Fig.(3)

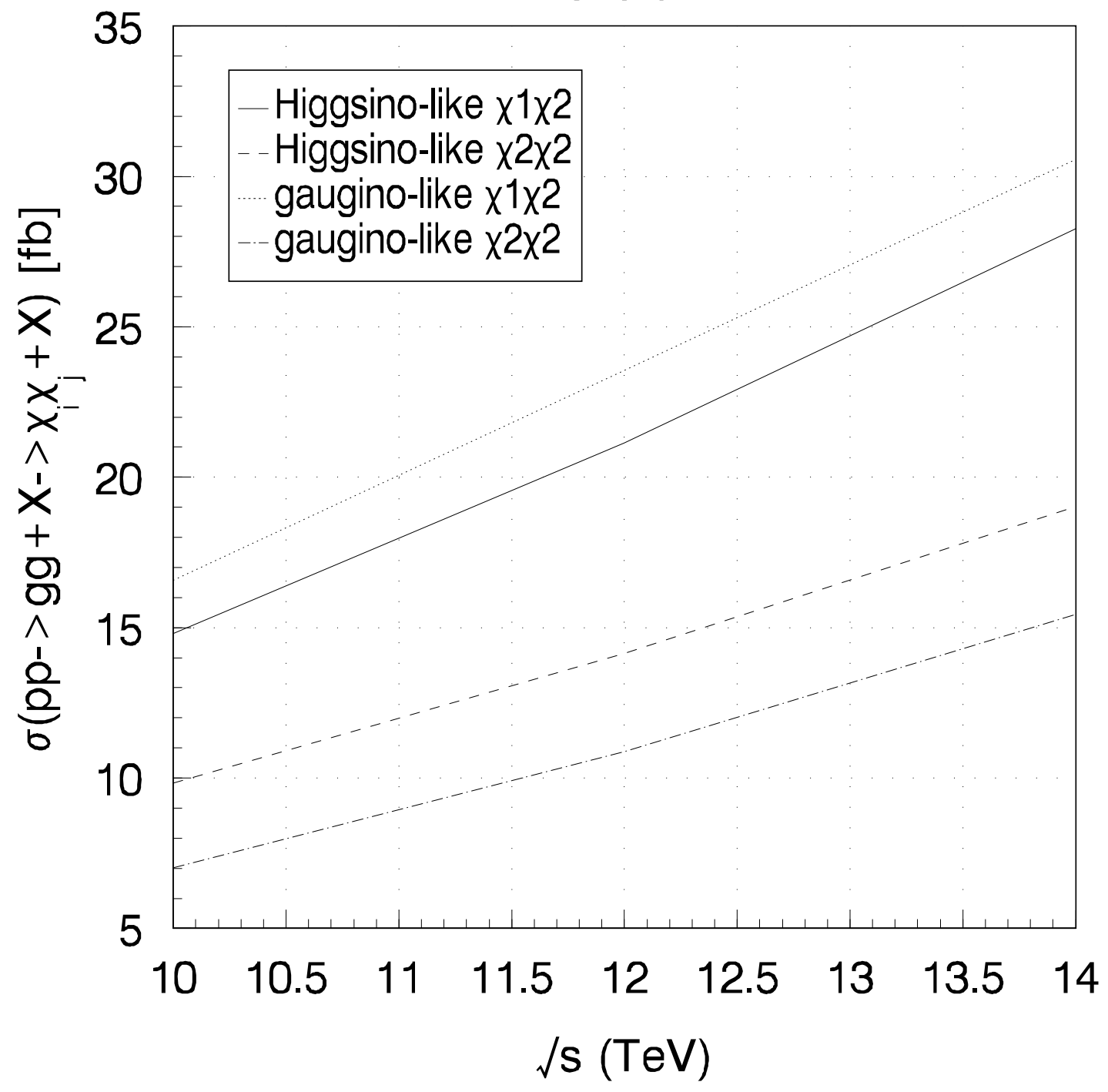




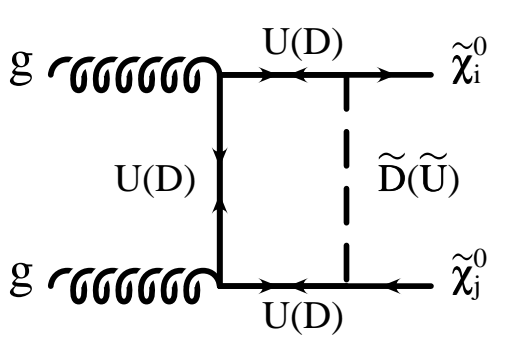

(a.1)

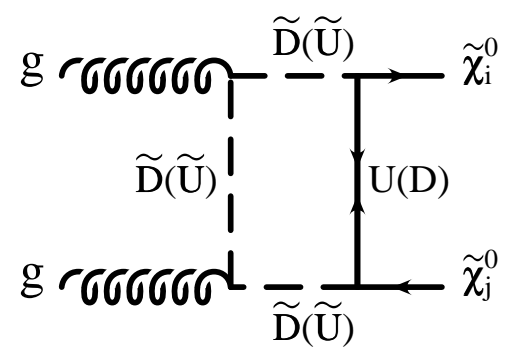

(a.2)

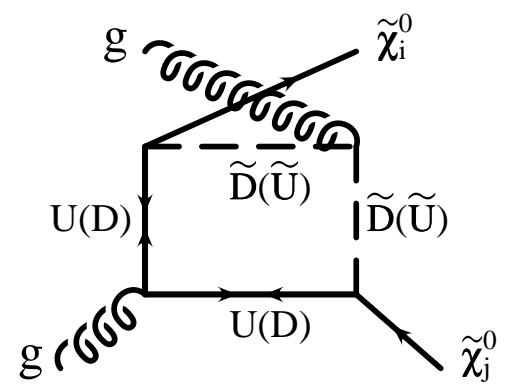

(a.3)

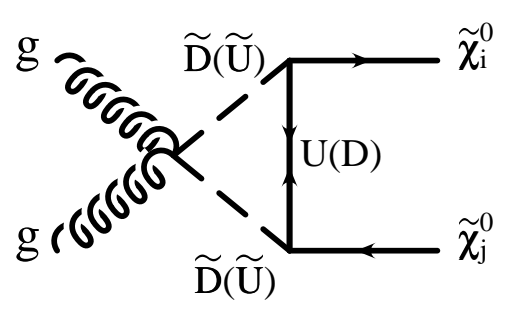

(b.1)

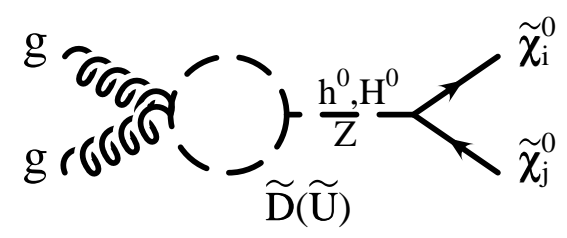

(b.2)

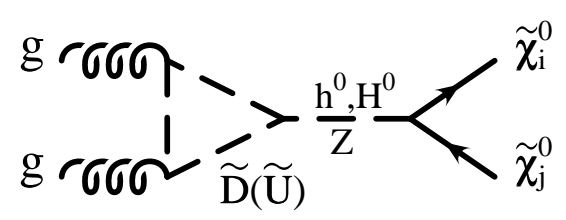

(c.1)

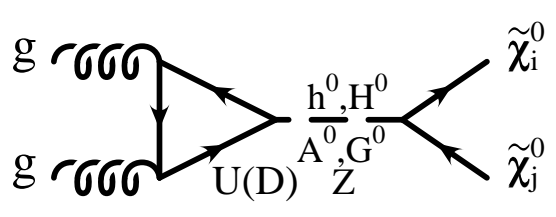

(c.2)

Fig.1 
Fig.(4)

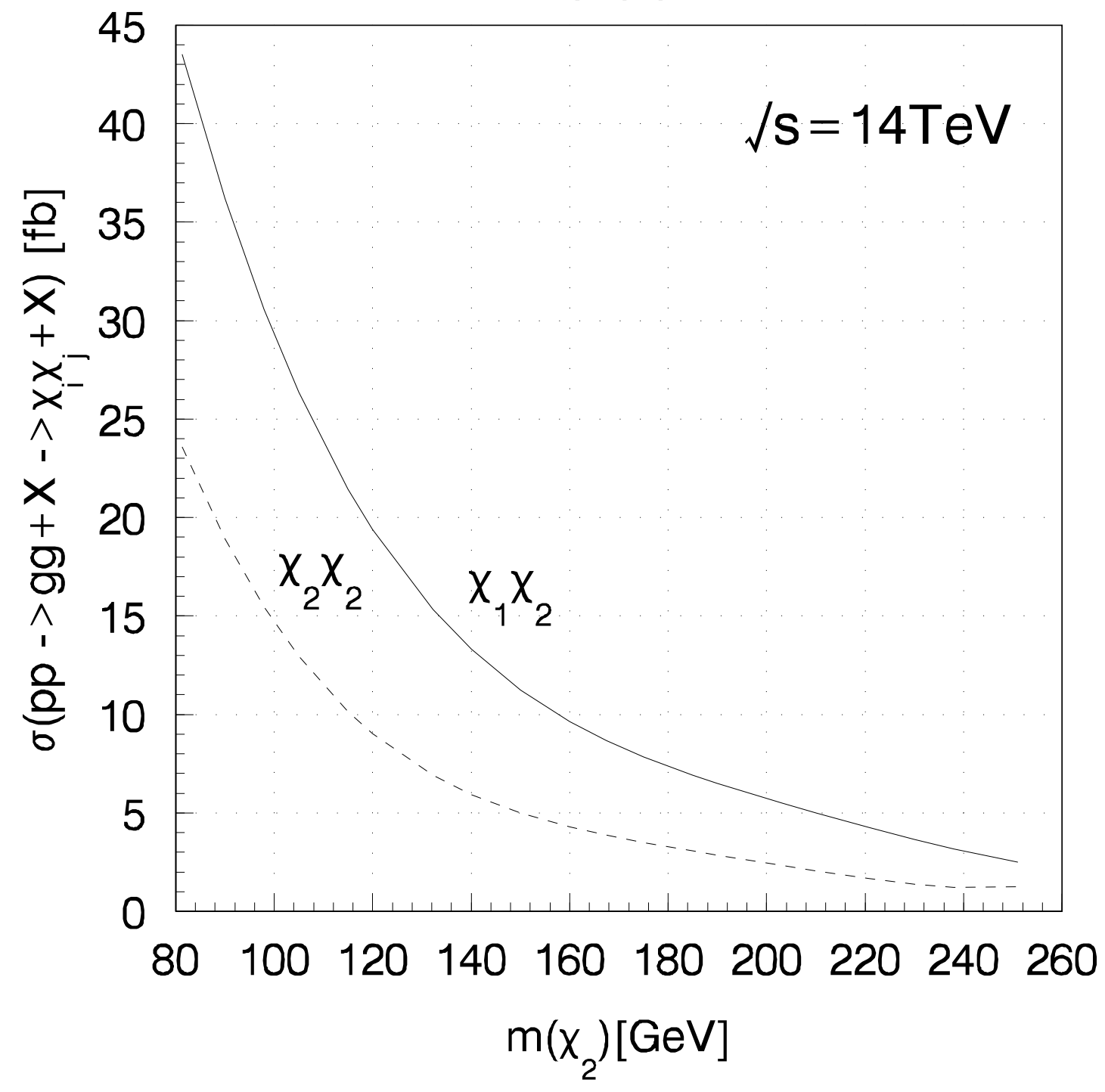




\title{
Pair production of neutralinos via gluon-gluon collisions *
}

\author{
Jiang $\mathrm{Yi}^{b}$, Ma Wen-Gan ${ }^{a, b}$, Han Liang ${ }^{b}$, Yu Zeng-Hui ${ }^{c}$ and H. Pietschmann ${ }^{c}$ \\ ${ }^{a}$ CCAST (World Laboratory), P.O.Box 8730, Beijing 100080, China. \\ ${ }^{b}$ Department of Modern Physics, University of Science and Technology \\ of China (USTC), Hefei, Anhui 230027, China. \\ ${ }^{c}$ Institut für Theoretische Physik, Universität Wien, A-1090 Vienna, Austria
}

\begin{abstract}
The production of a neutralino pair via gluon-gluon fusion is studied in the minimal supersymmetric model(MSSM) at proton-proton colliders. The numerical analysis of their production rates are carried out in the mSUGRA scenario. The results show that this cross section may reach about 80 femto barn for $\tilde{\chi}_{1}^{0} \tilde{\chi}_{2}^{0}$ pair production and 23 femto barn for $\tilde{\chi}_{2}^{0} \tilde{\chi}_{2}^{0}$ pair production with suitable input parameters at the future LHC collider. It shows that this loop mediated process can be competitive with the quark-antiquark annihilation process at the LHC.
\end{abstract}

PACS number(s): 14.80.Ly, 12.15.Ji, 12.60.Jv

\footnotetext{
${ }^{*}$ The project supported by National Natural Science Foundation of China
} 


\section{Introduction}

The Standard Model(SM) [1][2] is a successful theory of strong and electroweak interactions up to the present accessible energies. The hierarchy problem suggests that in principle the SM is the low energy effective theory of a more fundamental one. Supersymmetry (SUSY) is presently the most popular attempt to solve the hierarchy problem of the SM, where the cancellation of quadratic divergences is guaranteed and hence any mass scale is stable under radiative corrections. The most favorable candidate for a realistic extension of the SM is the minimal supersymmetric standard model(MSSM). In the MSSM, a discrete symmetry called R-parity [3] is kept in order to assure baryon and lepton number conservations, because the gauge-coupling unification supports conservation of R-parity $\llbracket$. It implies that there exists an absolutely stable lightest supersymmetric particle(LSP). Proper electroweak symmetry

breaking induces the right properties of the LSP being a natural weakly interacting cold dark matter candidate, which can explain many astrophysical observations [5]. In most cases the LSP in the MSSM may be the lightest Majorana fermionic neutralino. The neutralinos are mass eigenstates which are model dependent linear combinations of neutral gauginos and higgsinos [6]. They are determined by diagonalizing the corresponding mass matrix. In the MSSM the mass matrix depends on four unknown parameters, namely $\mu, M_{2}, M_{1}$, and $\tan \beta=v_{2} / v_{1}$, the ratio of the vacuum expectation values of the two Higgs fields. $\mu$ is the supersymmetric Higgs-boson-mass parameter and $M_{2}$ and $M_{1}$ are the gaugino mass parameters associated with the $S U(2)$ and $U(1)$ subgroups, respectively.

Direct search of supersymmetric particles in experiment is one of the promising tasks for 
present and future colliders. The multi-TeV Large Hadron Collider(LHC) at CERN and the possible future Next Linear Collider(NLC) are elaborately designed in order to study the symmetry-breaking mechanism and new physics beyond the SM. When the LEP2 running will be terminated, the hadron colliders Tevatron and LHC will be the machines left in searching for supersymmetric particles. Therefore it is necessary to give a proper and full understanding of the production mechanisms of supersymmetric particles at hadron colliders. If supersymmetry really exists at TeV scale, SUSY particles should be discovered and it will be possible to make accurate measurements to determine their masses and other parameters of the Lagrangian at the LHC, and then we will have a better understanding of the supersymmetric model[7]. We know that there are several mechanisms inducing the production of a chargino/neutralino pair at hadron colliders. One is through the quark-antiquark annihilation called Drell-Yan process, and another is via gluon-gluon fusion. Although the neutralino pair production via gluon-gluon fusion is a process induced by one-loop Feynman diagrams, the production rate can be still significant due to the large gluon luminosity in hadron colliders. The direct production channels of chargino/neutralinos and sleptons at the hadron colliders Tevatron and LHC $p \bar{p} / p p \rightarrow \tilde{\chi}_{i} \tilde{\chi}_{j}+X$ and $\tilde{l} \tilde{l}^{\prime}+X$ via quark-antiquark annihilation are investigated by Beenakker et al[8]. A recent work [12] showed that the one-loop process of the lightest chargino pair production via gluon-gluon fusion at the LHC can be considered as part of the NLO QCD correction to the quark-antiquark annihilation process. The neutralino pair production via quark-antiquark annihilation at the LHC was also considered by Han et al.[9]. The measurement of CP violating supersymmetric phases 
in the chargino and neutralino pair production at the NLC was investigated by Barger et al[10]. The lower mass limit of $29.1 \mathrm{GeV}$ on the lightest neutralino is obtained experimentally by analyzing the DELPHI results with the assumption that $M_{1} / M_{2} \gtrsim 0.5, \tan \beta=1$, $\mu=-62.3 \mathrm{GeV}$ and $M_{2}=46.0 \mathrm{GeV}[11]$.

In this paper we concentrate on the direct neutralino pair production via gluon-gluon collisions at the LHC in the framework of the MSSM with complete one-loop Feynman diagrams. The numerical calculation will be illustrated in the CP conserving mSUGRA scenario with five input parameters, namely $m_{1 / 2}, m_{0}, A_{0}, \mu$ and $\tan \beta$, where $m_{1 / 2}, m_{0}$ and $A_{0}$ are the universal gaugino mass, scalar mass at GUT scale and the trilinear soft breaking parameter in the superpotential respectively. From these five parameters, all the masses and couplings of the model are determined by the evolution from the GUT scale down to the low electroweak scale[13]. The paper is organized as follows: In section 2, we introduce the relevant features of the model and the analysis of the cross section in this work. In section 3, we discuss the numerical results of the cross sections and finally, a short summary is presented.

\section{The Calculation of $p p \rightarrow g g+X \rightarrow \tilde{\chi}_{i}^{0} \tilde{\chi}_{j}^{0}+X$}

In the MSSM the physical neutralino mass eigenstates $\tilde{\chi}_{i}^{0}(i=1,2,3,4)$ are the combinations of the neutral gauginos, $\tilde{B}$ and $\tilde{W}^{3}$, and the neutral higgsino, $\tilde{H}_{1}^{0}$ and $\tilde{H}_{2}^{0}$. In the two-component fermion fields $\psi_{j}^{0}=\left(-i \lambda^{\prime},-i \lambda^{3}, \psi_{H_{1}^{0}}, \psi_{H_{2}^{0}}\right)$ [6], where $\lambda^{\prime}$ is the bino and $\lambda^{3}$ is the neutral wino, the neutralino mass term in the Lagrangian is given by 


$$
\mathcal{L}_{M}=-\frac{1}{2}\left(\psi^{0}\right)^{T} Y \psi^{0}+\text { h.c. }
$$

where the matrix $Y$ reads

$$
Y=\left(\begin{array}{cccc}
M_{1} & 0 & -m_{Z} \sin \theta_{W} \cos \beta & m_{Z} \sin \theta_{W} \sin \beta \\
0 & M_{2} & m_{Z} \cos \theta_{W} \cos \beta & -m_{Z} \cos \theta_{W} \sin \beta \\
-m_{Z} \sin \theta_{W} \cos \beta & m_{Z} \cos \theta_{W} \cos \beta & 0 & -\mu \\
m_{Z} \sin \theta_{W} \sin \beta & -m_{Z} \cos \theta_{W} \sin \beta & -\mu & 0
\end{array}\right)
$$

$M_{1}, M_{2}$ and $\mu$ can be complex and introduce CP-violating phases. By reparametrization of the fields, $M_{2}$ can be real and positive without loss of generality. The matrix $Y$ is symmetric and can be diagonalized by one unitary matrix $N$ such that $N_{D}=N^{*} Y N^{+}=$ $\operatorname{diag}\left(m_{\tilde{\chi}_{1}^{0}}, m_{\tilde{\chi}_{2}^{0}}, m_{\tilde{\chi}_{3}^{0}}, m_{\tilde{\chi}_{4}^{0}}\right)$ with the order of $m_{\tilde{\chi}_{1}^{0}} \leq m_{\tilde{\chi}_{2}^{0}} \leq m_{\tilde{\chi}_{3}^{0}} \leq m_{\tilde{\chi}_{4}^{0}}$. Then the twocomponent mass eigenstates can be

$$
\chi_{i}^{0}=N_{i j} \psi_{j}^{0}, \quad i, j=1, \ldots, 4
$$

The proper four-component mass eigenstates are the neutralinos which are defined in terms of two-component fields as

$$
\tilde{\chi}_{i}^{0}=\left(\begin{array}{c}
\chi_{i}^{0} \\
\bar{\chi}_{i}^{0}
\end{array}\right) \quad(i=1, \ldots, 4),
$$

and the mass term becomes

$$
\mathcal{L}_{m}=-\frac{1}{2} \sum_{i} \tilde{M}_{i} \overline{\tilde{\chi}}_{i}^{0} \tilde{\chi}_{i}^{0},
$$

where $\tilde{M}_{i}$ are the diagonal elements of $N_{D}$.

The neutralino pair via gluon-gluon collisions can only be produced through one-loop diagrams in the lowest order. The calculation for this process can be simply carried out 
by summing all unrenormalized reducible and irreducible diagrams and the result will be finite and gauge invariant. In this work, we perform the calculation in the 't Hooft-Feynman gauge. The generic Feynman diagrams contributing to the subprocess $g g \rightarrow \tilde{\chi}_{i}^{0} \tilde{\chi}_{j}^{0}$ in the MSSM are depicted in Fig.1, where the exchange of incoming gluons in Fig.1(a.1 3) and Fig.1(c.1 $\sim 2)$ are not shown. All the one-loop diagrams can be divided into three groups: (1) box diagrams shown in Fig.1(a.1 3), (2) quartic interaction diagrams in Fig.1(b.1 2), (3) triangle diagrams shown in Fig.1(c.1 $\sim 2$ ). The $Z^{0}$ boson intermediated s-channel diagrams with quark or squark loops, as shown in Fig.1(b.2), Fig.1(c.1) and Fig.1(c.2), cannot contribute to the cross section. That can be explained in two fields. Firstly, the vector component of the $Z^{0}$ boson wave function does not couple to the initial gg state as the result of the Laudau-Yang theorem. Secondly, the CP-odd scalar component of the $Z^{0}$ boson does not couple to the invariant CP-even $\tilde{\chi}_{i}^{0} \tilde{\chi}_{j}^{0}$ state. We should mention that there are also some diagrams not contributing to the process, which we do not draw them in Fig.1(for example the s-channel diagrams with trilinear gluon interactions). Since the vertices of $A^{0}\left(G^{0}\right)-\tilde{q}-\tilde{q}$ vanish [14], there is no diagrams with a triangle squark loop coupling with an $A^{0}$ or $G^{0}$ Higgs boson.

We denote the reaction of neutralino pair production via gluon-gluon collision as:

$$
g\left(p_{3}, \mu\right) g\left(p_{4}, \nu\right) \longrightarrow \tilde{\chi}_{i}^{0}\left(p_{1}\right) \tilde{\chi}_{j}^{0}\left(p_{2}\right)
$$

The corresponding matrix element for each of the diagrams can be written as

$$
\mathcal{M}=\mathcal{M}^{\hat{s}}+\mathcal{M}^{\hat{t}}+\mathcal{M}^{\hat{u}}
$$


where $\mathcal{M}^{\hat{s}}, \mathcal{M}^{\hat{t}}$ and $\mathcal{M}^{\hat{u}}$ are the matrix elements from s-channel, t-channel and u-channel diagrams, respectively. The amplitude parts from the u-channel box and triangle vertex interaction diagrams can be obtained from the t-channel's by the following:

$$
\mathcal{M}^{\hat{u}}=(-1)^{\delta_{i j}} \mathcal{M}^{\hat{t}}\left(\hat{t} \rightarrow \hat{u}, p_{3} \leftrightarrow p_{4}, \mu \leftrightarrow \nu\right),
$$

where the indices $\mathrm{i}$ and $\mathrm{j}$ are for the final neutralino. When $i=j$, there is a relative minus sign due to the Fermi statistics, which requires the amplitude to be antisymmetric under interchange of the two final identical fermions. The cross section for this subprocess at one-loop order in unpolarized gluon collisions can be obtained by

$$
\hat{\sigma}\left(\hat{s}, g g \rightarrow \tilde{\chi}_{i}^{0} \tilde{\chi}_{j}^{0}\right)=\frac{1}{16 \pi \hat{s}^{2}}\left(\frac{1}{2}\right)^{\delta_{i j}} \int_{\hat{t}^{-}}^{\hat{t}^{+}} d \hat{t} \sum^{-}|\mathcal{M}|^{2}
$$

where $\hat{t}^{ \pm}=1 / 2\left[\left(m_{\tilde{\chi}_{i}}^{2}+m_{\tilde{\chi}_{j}}^{2}-\hat{s}\right) \pm \sqrt{\left(m_{\tilde{\chi}_{i}}^{2}+m_{\tilde{\chi}_{j}}^{2}-\hat{s}\right)^{2}-4 m_{\tilde{\chi}_{i}}^{2} m_{\tilde{\chi}_{j}}^{2}}\right]$. The factor $\left(\frac{1}{2}\right)^{\delta_{i j}}$ is due to the two identical particles in the final states. The bar over the sum means average over initial spins and colors.

With the results from Eq.(2.9), we can easily obtain the total cross section at $p p$ collider by folding the cross section of the subprocess $\hat{\sigma}\left(g g \rightarrow \tilde{\chi}_{i}^{0} \tilde{\chi}_{j}^{0}\right)$ with the gluon luminosity,

$$
\sigma\left(p p \rightarrow g g+X \rightarrow \tilde{\chi}_{i}^{0} \tilde{\chi}_{j}^{0}+X\right)=\int_{\left(m_{\tilde{\chi}_{i}^{0}}+m_{\tilde{\chi}_{j}^{0}}\right)^{2} / s}^{1} d \tau \frac{d \mathcal{L}_{g g}}{d \tau} \hat{\sigma}\left(g g \rightarrow \tilde{\chi}_{i}^{0} \tilde{\chi}_{j}^{0}, \quad \text { at } \hat{s}=\tau s\right)
$$

where $\sqrt{s}$ and $\sqrt{\hat{s}}$ denote the proton-proton and gluon-gluon c.m.s. energies respectively and $\frac{d \mathcal{L}_{g g}}{d \tau}$ is the gluon luminosity, which is defined as

$$
\frac{d \mathcal{L}_{g g}}{d \tau}=\int_{\tau}^{1} \frac{d x_{1}}{x_{1}}\left[f_{g}\left(x_{1}, Q^{2}\right) f_{g}\left(\frac{\tau}{x_{1}}, Q^{2}\right)\right]
$$


Here we used $\tau=x_{1} x_{2}$, the definitions of $x_{1}$ and $x_{2}$ are from Ref.[15]. In our numerical calculation we adopt the MRST(mode 2) parton distribution function $f_{g}\left(x_{i}, Q^{2}\right)$ [16], and ignore the supersymmetric QCD corrections to the parton distribution functions for simplicity. The

factorization scale $Q$ was chosen as the average of the final particle masses $\frac{1}{2}\left(m_{\tilde{\chi}_{i}}+m_{\tilde{\chi}_{j}}\right)$. The numerical calculation is carried out for the LHC at the energy $14 \mathrm{TeV}$.

\section{Numerical results and discussions}

In this section, we present some numerical results of the total cross section from the full one-loop diagrams involving virtual (s)quarks for the process $p p \rightarrow g g+X \rightarrow \tilde{\chi}_{i}^{0} \tilde{\chi}_{j}^{0}+X$, respectively. The input parameters are chosen as: $m_{t}=173.8 \mathrm{GeV}, m_{Z}=91.187 \mathrm{GeV}$, $m_{b}=4.5 \mathrm{GeV}, \sin ^{2} \theta_{W}=0.2315$, and $\alpha=1 / 128$. We adopt a simple one-loop formula for the running strong coupling constant $\alpha_{s}$

$$
\alpha_{s}(\mu)=\frac{\alpha_{s}\left(m_{Z}\right)}{1+\frac{33-2 n_{f}}{6 \pi} \alpha_{s}\left(m_{Z}\right) \ln \left(\frac{\mu}{m_{Z}}\right)} .
$$

where $\alpha_{s}\left(m_{Z}\right)=0.117$ and $n_{f}$ is the number of active flavors at energy scale $\mu$. In our numerical calculation to get the low energy scenario from the mSUGRA, the renormalization group equations(RGE's) [17] are run from the weak scale $M_{Z}$ up to the GUT scale, taking all threshold into account. We use two loop RGE's only for the gauge couplings and the oneloop RGE's for the other supersymmetric parameters. The GUT scale boundary conditions are imposed and the RGE's are run back to $M_{Z}$, again taking threshold into account. The decay widths of the intermediate Higgs bosons are considered at the tree level and these formula can be found in ref.[18]. 
The neutralino pair production cross sections dependence on the factorization scale Q is illustrated in Fig.2, where we choose the mSUGRA parameters as $m_{0}=100 \mathrm{GeV}, m_{1 / 2}=$ $150 \mathrm{GeV}, A=300 \mathrm{GeV}, \tan \beta=4$ and $\mu>0$. The masses of $\tilde{\chi}_{1}^{0} \tilde{\chi}_{2}^{0}$ are $51.4 G e V$ and $98 G e V$. For the $\tilde{\chi}_{1}^{0} \tilde{\chi}_{2}^{0}$ pair production, the cross section changes from $28 f b$ to $30 f b$ when the scale $\mathrm{Q}$ changes from $0.2 m_{\tilde{x}_{2}^{0}}$ to $m_{\tilde{x}_{2}^{0}}$. For the $\tilde{\chi}_{2}^{0} \tilde{\chi}_{2}^{0}$ pair production, the cross section changes from $14 f b$ to $15.5 f b$ when the $\mathrm{Q}$ changes from $0.2 m_{\tilde{x}_{2}^{0}}$ to $m_{\tilde{x}_{2}^{0}}$. When $\mathrm{Q}$ is larger than $m_{\tilde{x}_{2}^{0}}$, the cross sections of these two processes are nearly independent of the factorization scale.

In Fig.3 we show the cross sections of gaugino-like neutralino pair production and the higgsino-like neutralino pair production. For the gaugino-like neutralino pair production, we choose the same values of mSUGRA parameters adopted in Fig.2. But in the higgsino-like neutralino case, we choose $M_{1}=150 \mathrm{GeV}, M_{2}=210 \mathrm{GeV}$ and $\mu=90 \mathrm{GeV}$ in order to keep the masses of $\tilde{\chi}_{1}^{0}$ and $\tilde{\chi}_{2}^{0}$ being the same as those in the previous gaugino-like neutralino case, the other parameters of the higgsino-like neutralino case at the weak scale are chosen being the same as those in the gaugino-like neutralino case. Then the difference of the cross sections between gaugino-like neutralino pair production and higgsino-like neutralino pair production only comes from the change of the coupling of $\tilde{\chi}_{i}^{0}-\tilde{q}-q$ and $\tilde{\chi}_{i}^{0}-\tilde{\chi}_{j}^{0}-H^{0}\left(A^{0}, h^{0}, G^{0}\right)$ where the matrix elements of $\mathrm{N}$ are changed. For the $\tilde{\chi}_{1}^{0} \tilde{\chi}_{2}^{0}$ pair production, the cross section in the higgsino-like case is smaller than that in the gaugino-like case, but for the $\tilde{\chi}_{2}^{0} \tilde{\chi}_{2}^{0}$ pair production, the cross section in the higgsino-like case is larger than that in the gaugino-like case.

The cross sections for $\tilde{\chi}_{1}^{0} \tilde{\chi}_{2}^{0}$ and $\tilde{\chi}_{2}^{0} \tilde{\chi}_{2}^{0}$ productions at the LHC versus the mass of $\tilde{\chi}_{2}^{0}$ is 
shown in Fig.4. We assume that $\tilde{\chi}_{1}^{0}$ is the LSP and escapes detection. The input parameters are chosen as $m_{0}=100 \mathrm{GeV}, A_{0}=300 \mathrm{GeV}, \tan \beta=4, \mu>0$ and $m_{1 / 2}$ varying from $130 \mathrm{GeV}$ to $325 \mathrm{GeV}$. In the framework of the mSUGRA, the masses of $\tilde{\chi}_{1}^{0}$ and $\tilde{\chi}_{2}^{0}$ increase from $41 \mathrm{GeV}$ to $129 \mathrm{GeV}$ and from $81 \mathrm{GeV}$ to $251 \mathrm{GeV}$ respectively, and the masses of Higgs boson $\mathrm{H}^{0}$ and $A^{0}$ increase from $230 \mathrm{GeV}$ to $509 \mathrm{GeV}$ and from $226 \mathrm{GeV}$ to $507 \mathrm{GeV}$ respectively with our input parameters. In our case, the mass of the Higgs boson $h^{0}$ is always smaller than $m_{\tilde{\chi}_{1}^{0}}+m_{\tilde{\chi}_{2}^{0}}$ and the contribution to the cross section of $h^{0}$ is very small due to the s-channel suppression. Since the masses of $H^{0}$ and $A^{0}$ are larger than $m_{\tilde{\chi}_{1}^{0}}+m_{\tilde{\chi}_{2}^{0}}$, the cross section will be strongly enhanced by the s-channel resonance effects when the total energy $\sqrt{\hat{s}}$ of the subprocess approaches the mass of $H^{0}$ or $A^{0}$. Since $m_{\tilde{\chi}_{2}^{0}}>m_{\tilde{\chi}_{1}^{0}}$, the pair production of $\tilde{\chi}_{2}^{0} \tilde{\chi}_{2}^{0}$ compared to $\tilde{\chi}_{1}^{0} \tilde{\chi}_{2}^{0}$ is kinematically suppressed. For the $\tilde{\chi}_{1}^{0} \tilde{\chi}_{2}^{0}$ pair production, the cross section can reach 43 femto barn when $m_{1 / 2}=130 \mathrm{GeV}, m_{\tilde{\chi}_{1}^{0}}=41 \mathrm{GeV}$ and $m_{\tilde{\chi}_{2}^{0}}=81 \mathrm{GeV}$. The cross section of $\tilde{\chi}_{2}^{0} \tilde{\chi}_{2}^{0}$ pair production can reach 23 femto barn when $m_{1 / 2}=130 \mathrm{GeV}$. From the results of [8], we know that the cross sections of $\tilde{\chi}_{1}^{0} \tilde{\chi}_{2}^{0}$ and $\tilde{\chi}_{2}^{0} \tilde{\chi}_{2}^{0}$ pair production via quark-antiquark annihilation at the LHC are about 70 femto barn and 300 femto barn respectively, when the input parameters are chosen as $m_{0}=150 \mathrm{GeV}, A_{0}=300 \mathrm{GeV}$, $\tan \beta=4, \mu>0$ and $m_{\tilde{\chi}_{2}^{0}}=81 \mathrm{GeV}$. The pair production rate of $\tilde{\chi}_{1}^{0} \tilde{\chi}_{2}^{0}$ via gluon-gluon fusion is about few ten percent of that via quark-antiquark annihilation at the LHC with the same input parameters. The cross section of $\tilde{\chi}_{2}^{0} \tilde{\chi}_{2}^{0}$ via gluon-gluon fusion is about few percent of that via quark-antiquark annihilation at the LHC. The neutralino pair production via gluon-gluon fusion at the LHC is of the same order of the next leading order(NLO) 
QCD correction to the quark-antiquark production process. The neutralino pair production rate via gluon-gluon fusion should be considered in studying the pair pair productions of neutralinos at proton-proton colliders.

In Fig. 5 we present the cross sections of neutralino pair productions versus $\tan \beta$ where the other four input parameters are chosen as $m_{0}=100 \mathrm{GeV}, A_{0}=300 \mathrm{GeV}, \mu>0$ and $m_{1 / 2}=150 \mathrm{GeV}$. When $\tan \beta$ increases from 4 to 32 , the mass of $\tilde{\chi}_{1}^{0}$ increases from $51 \mathrm{GeV}$ to $57 \mathrm{GeV}$ and the mass of $\tilde{\chi}_{2}^{0}$ increases from $98 \mathrm{GeV}$ to $102.5 \mathrm{GeV}$. So the masses of neutralinos are nearly independent of $\tan \beta$. The masses of Higgs boson $H^{0}$ and $A^{0}$ depend on $\tan \beta$ and decrease from $258 \mathrm{GeV}$ to $165 \mathrm{GeV}$ and from $254 \mathrm{GeV}$ to $165 \mathrm{GeV}$ respectively. The mass of $h^{0}$ is a function of $\tan \beta$ too, but keeps $m_{h^{0}}<m_{\tilde{\chi}_{1}^{0}}+m_{\tilde{\chi}_{2}^{0}}$. Since the couplings of Higgs bosons to quarks and squarks pair are related to the ratio of the vacuum expectation values, $\tan \beta$ should effect the cross sections substantially. The cross sections of the neutralino pair productions decrease when $\tan \beta$ goes from 4 to 6 , and increase when $20>\tan \beta>6$ due to the effect of the coupling strength of Higgs bosons to quarks and squarks pair. For the $\tilde{\chi}_{1}^{0} \tilde{\chi}_{2}^{0}$ pair production, when $\tan \beta<27$, the masses of $H^{0}$ and $A^{0}$ are larger than $m_{\tilde{\chi}_{1}^{0}}+m_{\tilde{\chi}_{2}^{0}}$, the s-channel resonance effects of $H^{0}$ and $A^{0}$ on the cross section can be enhanced, while when $\tan \beta>27$, the masses of $H^{0}$ and $A^{0}$ become smaller than $m_{\tilde{\chi}_{1}^{0}}+m_{\tilde{\chi}_{2}^{0}}$, then there will be no s-channel resonance effects and the cross section goes down rapidly. For the similar reason, the cross section of the $\tilde{\chi}_{2}^{0} \tilde{\chi}_{2}^{0}$ pair production increase in the range of $20>\tan \beta>6$ due to the effect of the Yukawa couplings of Higgs bosons to quarks and squarks pair and the s-channel resonance effects of the intermediate Higgs bosons, but it goes down again when 
$\tan \beta>20$ where the masses of $H^{0}$ and $A^{0}$ become smaller than $m_{\tilde{\chi}_{2}^{0}}+m_{\tilde{\chi}_{2}^{0}}$ and the s-channel resonance effects of the Higgs bosons disappear. From the figure we can see that if we choose suitable input parameters, the cross section can be largely enhanced. Especially the cross section of the $\tilde{\chi}_{1}^{0} \tilde{\chi}_{2}^{0}$ pair production can even reach about 80 femto barn when $\tan \beta \sim 27$.

In our calculation we find that the discrepancy between our results from the newer sets of gluon densities in the MRST(mode 2) scheme and the older sets in the MRS(set G) scheme 19] is very small and cannot be distinguished in figures.

\section{Summary}

In this paper, we studied the pair production process of the neutralino via gluon-gluon fusion at the LHC collider. The numerical analysis of their production rates is carried out in the mSUGRA scenario with some typical parameter sets. The results show that the cross section of the neutralino pair-production via gluon-gluon fusion can reach about 80 femto barn for $\tilde{\chi}_{1}^{0} \tilde{\chi}_{2}^{0}$ pair production and 23 femto barn for $\tilde{\chi}_{2}^{0} \tilde{\chi}_{2}^{0}$ pair production at the LHC collider. In some c.m.s energy regions of incoming gluons, where the s-channel resonance conditions are satisfied in the parameter space, we can see observable enhancement effects of the cross sections. We conclude that neutralino pair production via gluon-gluon fusion can be competitive with the quark-antiquark annihilation production process at the LHC and should be considered as a part of the NLO QCD correction to the quark-antiquark annihilation production process.

Acknowledgement: This work was supported in part by the National Natural Science 
Foundation of China(project number: 19875049), the Youth Science Foundation of the University of Science and Technology of China, a grant from the Education Ministry of China

and the exchange program between China and Austria(Project number V.A.12). One of the authors Y. Jiang would like to thank Prof. F.F. Schöberl for valuable discussion in this context.

\section{References}

[1] S.L. Glashow, Nucl. Phys. 22(1961)579; S. Weinberg, Phys. Rev. Lett. 1(1967)1264; A. Salam, Proc. 8th Nobel Symposium Stockholm 1968, ed. N. Svartholm(Almquist and Wiksells, Stockholm 1968) p.367; H.D. Politzer, Phys. Rep. 14(1974)129.

[2] P.W. Higgs, Phys. Lett 12(1964)132, Phys. Rev. Lett. 13 (1964)508; Phys.Rev. 145(1966)1156; F.Englert and R.Brout, Phys. Rev. Lett. 13(1964)321; G.S. Guralnik, C.R.Hagen and T.W.B. Kibble, Phys. Rev. Lett. 13(1964)585; T.W.B. Kibble, Phys. Rev. 155(1967)1554.

[3] J. Ellis, et al., Nucl. Phys. B238, 53(1984).

[4] S. P. martin, Phys. Rev. D46, 2769(1992); E. Diehl, G.L. Kane, C. Kolda and J. D. Wells, Phys. Rev. D52, 4223(1993)

[5] R. Davis, et al., Phys. Rev. Lett. 20, 1205(1968); K. S. Hirata, et al., Phys. Rev. Lett. 65, 1297(1990); P. Anselmann, et al., Phys. Lett. B327, 377(1994); P.Sikivie, 
in Trends in Astroparticle Physics, Proceeding of the Workshop, Stockholm, Sweden, 1994, edited by L. Bergstrom et al..

[6] H.E. Haber and G.L. Kane, Phys. Rep. 117(1985)75.

[7] C.X. Chang, L. Han, W.G. Ma et al Nucl. Phys. B515 (1998)15; W. Beenakker, M. Krammer, et al Nucl. Phys. B515(1998)3.

[8] W. Beenakker, et al., Phys. Rev. Lett. 83 (1999)3780.

[9] Han Liang, Ma Wen-Gan, Jiang Yi, Zhou Mian-Lai and Zhou Hong, accepted by Commun. Theor. Phys.

[10] V. Barger, T. Han, T Li and T. Plehn, hep-ph/9907425.

[11] P. Abreu, et al., Phys. Lett. B446, 75(1999).

[12] Ma Wen-Gan, Du Fei, Zhou Mian-Lai, Jiang Yi, Han Liang and Zhou Hong, Phys. Rev. D60, 115009.

[13] M. Drees and S. P. Matrin, hep-ph/9504324.

[14] J. F. Gunion and H. E. Haber, Nucl. Phys. B272, 1(1986).

[15] Jiang Yi, et al, J. Phys. G23, 385(1997); G24, 83(E)(1998).

[16] A. D. Martin, R. G. Roberts, W. J. Stirling and R. S. Thorne, hep-ph/9907231.

[17] V. Barger, M. S. Berger and P. Ohmann, Phys. Rev. D47, 1093(1993), D47, 2038(1993); V. Barger, M. S. Berger, P. Ohmann and R. J. N. Phillips, Phys. 
Lett. B314, 351(1993); V. Barger, M. S. berger and P. Ohmann, Phys. Rev. D49, 4908(1994).

[18] J. Gunion, H. Haber, G. Kane and S. Dawson, The Higgs Hunter's Guide (AddisonWesley, Reading 1990).

[19] A. D. Martin, W. J. Stirling and R. G. Roberts, Phys. Lett. B354, 155(1995).

\section{Figure Captions}

Fig. 1 The Feynman diagrams of the subprocess $g g \rightarrow \tilde{\chi}_{i}^{0} \tilde{\chi}_{j}^{0}$.

Fig.2 Dependence of the cross sections for the productions of $\tilde{\chi}_{1}^{0} \tilde{\chi}_{2}^{0}$ and $\tilde{\chi}_{2}^{0} \tilde{\chi}_{2}^{0}$ pairs on the factorization scale Q with the mSUGRA parameters $m_{0}=100 \mathrm{GeV}, m_{1 / 2}=150 \mathrm{GeV}$, $A=300 \mathrm{GeV}, \tan \beta=4$ and $\mu>0$. The masses of $\tilde{\chi}_{1}^{0} \tilde{\chi}_{2}^{0}$ are $51.4 \mathrm{GeV}$ and $98 \mathrm{GeV}$.

Fig.3 The cross sections for the neutralino pair production at the gaugino-like neutralino case and higgsino-like neutralino case at the LHC. For the gaugino-like neutralino case, the parameters are chosen in mSUGRA scenario with $m_{0}=100 \mathrm{GeV}, m_{1 / 2}=150 \mathrm{GeV}$, $A=300 \mathrm{GeV}, \tan \beta=4$ and $\mu>0$. For the higgsino-like neutralino case, we choose $M_{1}=150 \mathrm{GeV}, M_{2}=210 \mathrm{GeV}, \mu=90 \mathrm{GeV}$ and the other parameters the same as those in the gaugino-like neutralino case.

Fig.4 Total cross sections of the process $p p \rightarrow g g+X \rightarrow \tilde{\chi}_{i}^{0} \tilde{\chi}_{j}^{0}+X$ as function of $m_{\tilde{\chi}_{2}^{0}}$ at the LHC. The solid curve is for $\tilde{\chi}_{1}^{0} \tilde{\chi}_{2}^{0}$ pair production and the dashed curve is for $\tilde{\chi}_{2}^{0} \tilde{\chi}_{2}^{0}$ pair production. 
Fig.5 Total cross sections of the process $p p \rightarrow g g+X \rightarrow \tilde{\chi}_{i}^{0} \tilde{\chi}_{j}^{0}+X$ as function of $\tan \beta$ at the LHC. The solid curve is for $\tilde{\chi}_{1}^{0} \tilde{\chi}_{2}^{0}$ pair production and the dashed curve is for $\tilde{\chi}_{2}^{0} \tilde{\chi}_{2}^{0}$ pair production. 
Fig.(2)

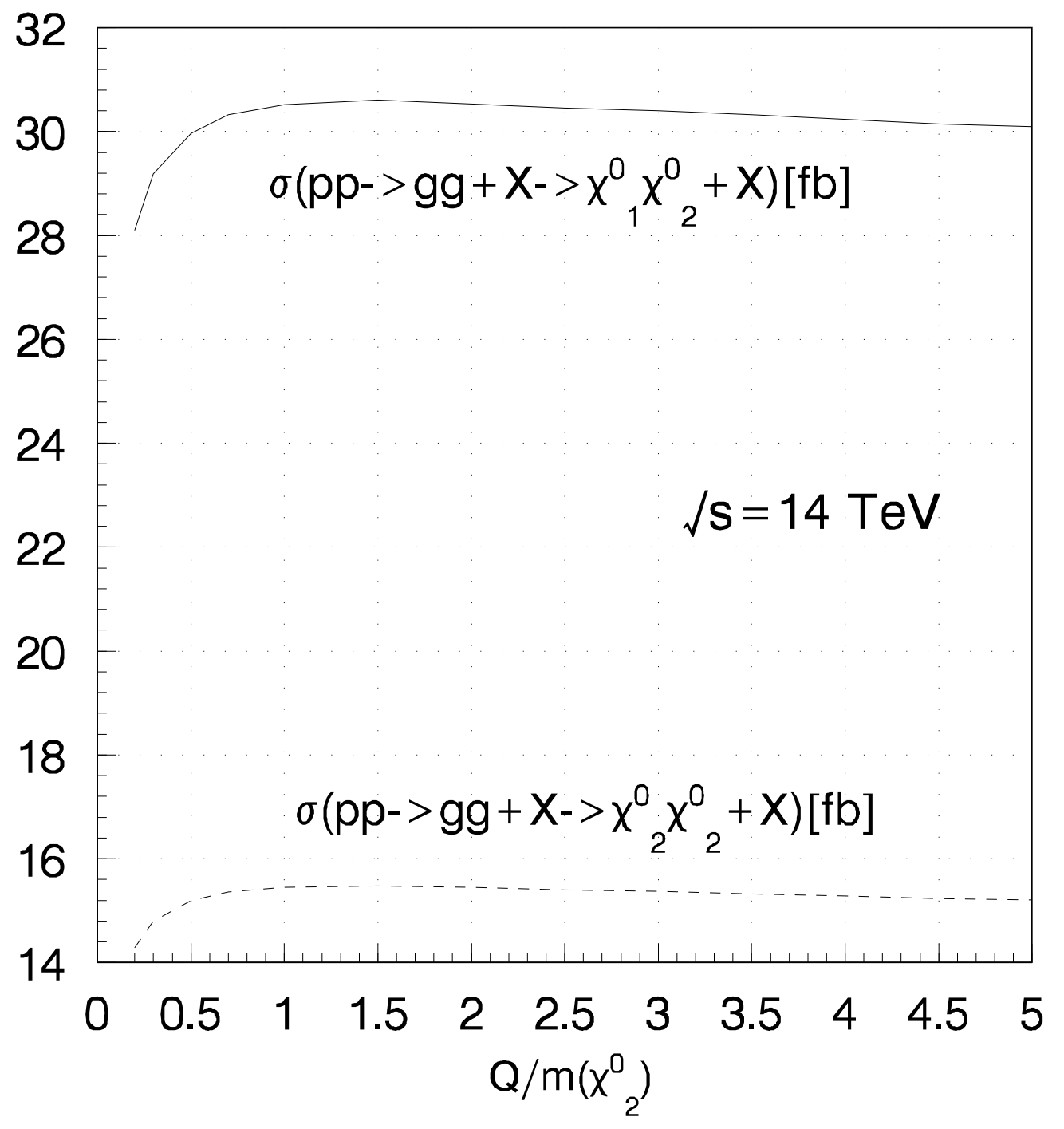


Fig.(5)

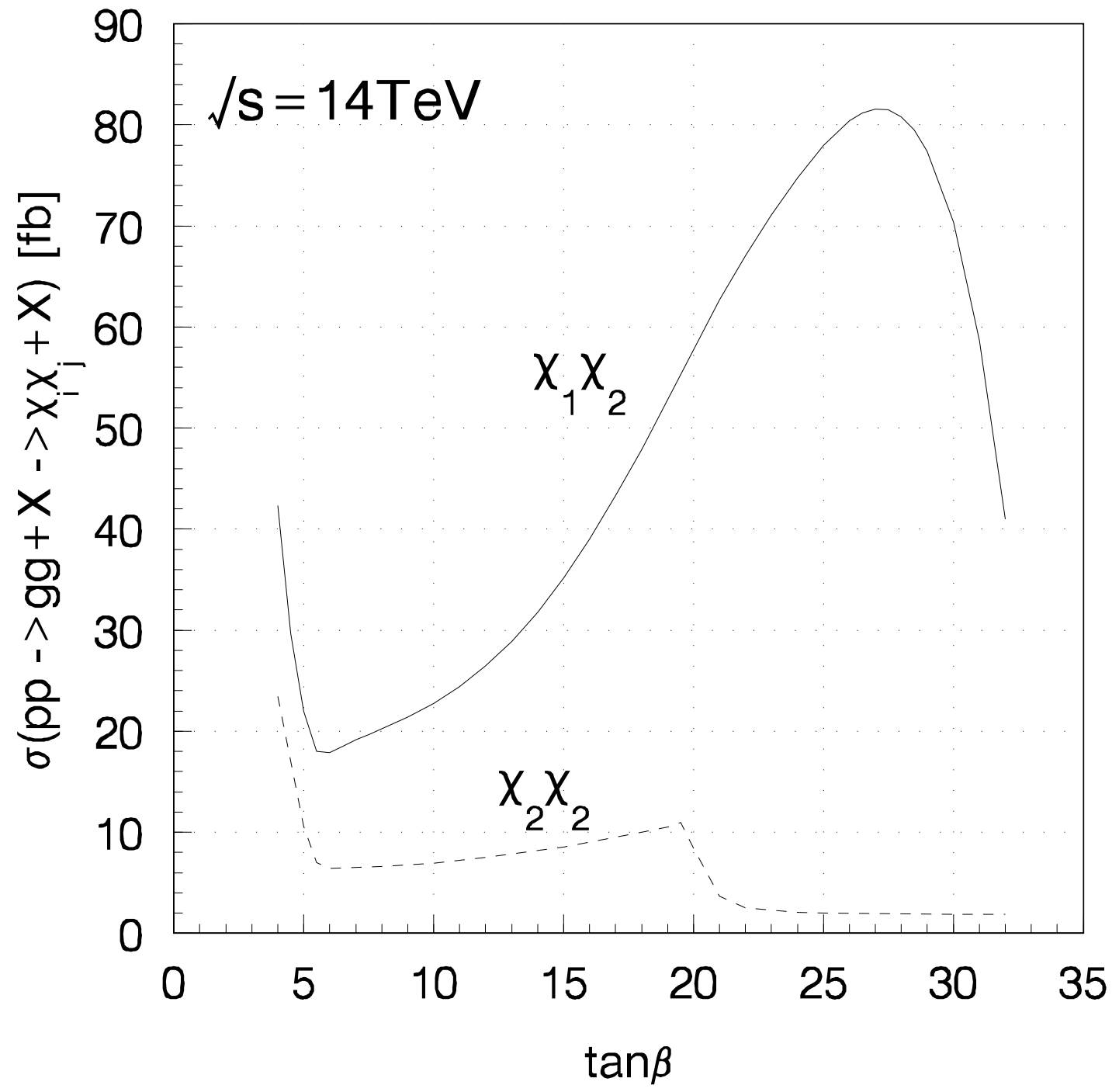

